\title{
Grid-based Localization and Mapping Method without Odometry Information
}

\author{
Kazuaki Okada \\ Department of Electrical and Computer Engineering \\ Yokohama National University \\ Tel:+81-45-339-4174 \\ Email: kazuaki@fujilab.dnj.ynu.ac.jp
}

\author{
Yasutaka Fujimoto \\ Department of Electrical and Computer Engineering \\ Yokohama National University \\ Tel:+81-45-339-4175 \\ Email: fujimoto@ynu.ac.jp
}

\begin{abstract}
In this paper, we propose a new localization and mapping method using the gridded map and range scan data only. The proposed method is applied to the autonomous wheelchair system. In this method we applied Particle Swarm Optimization (PSO) with appropriate initial values to estimate displacements of position and orientation of the wheelchair. We compared a conventional method that used cross correlation from a previous scan data and a current scan data and our method in some experiments. The experimental results in four situations show the high accuracy estimation and high processing speed of our method.
\end{abstract}

\section{INTRODUCTION}

Robots are used not only in the industrial field but also in the living environment. For example, autonomous mobile cleaning robots are available in homes. Development and research of the navigation robot which can work together in various environments are widely conducted. If these technologies can be extended to the electric wheelchair, it becomes very useful system.

The electric wheelchair is an important vehicle for aged and handicapped people. However, it is reported that sometimes accident occurs attributing to the operation mistake. Autonomous navigation system can decrease some accident. It will enable the electric wheelchair controlled more easily and safely. One of system was already developed [1], which has many sensors and uses its odometory information. For such systems, it is doubtless that implementation of the simultaneous localization and map building (SLAM) is the most important problem. This problem is solved by using a

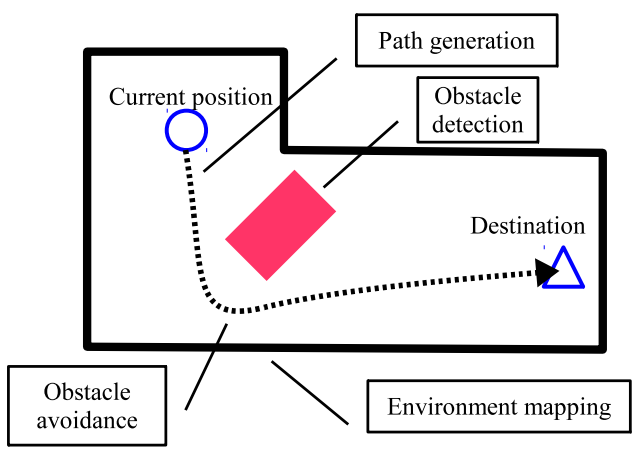

Fig. 1. Robot navigation positional data and environmental data from the range sensor. Some algorithm was proposed for this problem. The most popular method of SLAM is FastSLAM [2]. This method needs the geometric feature in the scan data and the odometry information. The mapping method is used on the rescue robot [3]. These robots saves various information, because it detects various objects reliably. These maps are saved as 3D information.

The geometric feature based method is one of algorithm of the SLAM [4]. It calculates cross correlation between a previous scan data and a current scan data. This method uses only scan data from the range sensor and calculation cost is low. However its solution is a discrete value. Iterative Closest Point (ICP) Algorithm [5] is the most popular matching method based on geometric feature for SLAM problem. This method needs geometric features from a scan data. Many methods obtain solution by iterative calculation. There are various approaches in the optimization of the ICP [6]. The ICP method uses the laser reflectance intensity [7]. This method can use some particular sensors, which return values of range and reflectance intensity. Additionally, the competition of SLAM called "Tsukuba Challenge" attracts many interests of researchers in this field. Many robots are developed for this challenge. Many robots use scan data of certain height level in out-doors [8]. These methods are not applicable in the in-door environment.

Hence we proposed new electric wheelchair system with simple structure. It solves SLAM problem using few sensors. Our method can estimate position, orientation and map of the environment both in doors and in out-doors that is confirmed by some experiments [10]. This paper presents our improved system and its evaluation of its accuracy by four experiments.

The rest of the paper is organized as follows: Section II describes our system structure and proposed method for estimation of the position and the orientation using PSO. We contrast between proposed method and conventional method in Section III. Finally, in Section IV we conclude and describe future work.

\section{SYSTEM STRUCTURE}

Our system is shown in the Fig. 2. It is made of an electric wheelchair as controlled object and a computer equipped on the wheelchair as controller. The range sensor is set on the 


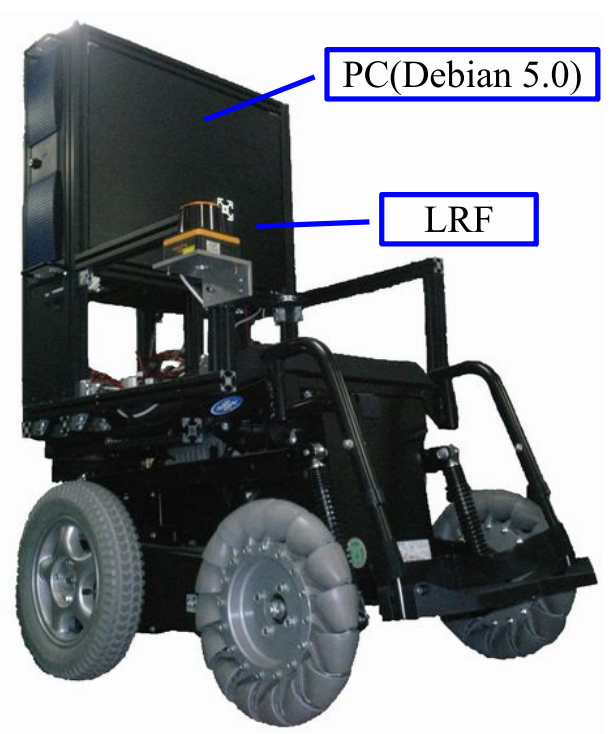

Fig. 2. Picture of an autonomous wheel chair

right side of the whole machine, which is used to get the location data in the horizontal plane. While the wheelchair is moving, the computer will draw the map of the space in the monitor with the data scanned by a range sensor to show an operator the location of obstacle.

Various sensors are applied to SLAM, such as the Laser Range Finder (LRF), Global Positioning System (GPS), camera, magnetic sensor and so on. Since some sensors such as GPS do not work indoors, we adopt the LRF as the scan sensors, which is more suitable for our system. The LRF used in our research is the "UHG-08LX" produced by HOKUYO AUTOMATIC CO.LTD, which has $8 \mathrm{~m}$ radius range with a measuring accuracy of $1 \mathrm{~mm}$ and $270 \mathrm{deg}$ angular range that means an angular resolution of $360 \mathrm{deg} / 1024=0.3515625$ deg. The Scan time is about $67 \mathrm{msec} / \mathrm{scan}(14.925 \mathrm{~Hz})$. The distances are scanned point by point from the left to the right. Additionally, the accuracy of this LRF is $3 \%$

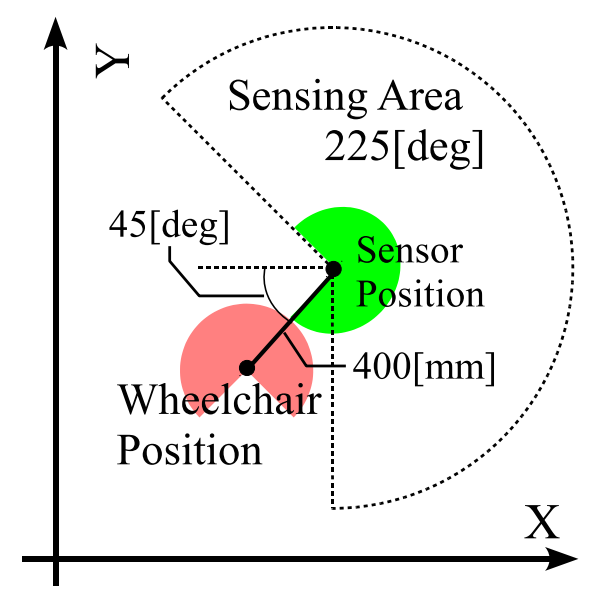

Fig. 3. Position relation and sensing area of LRF

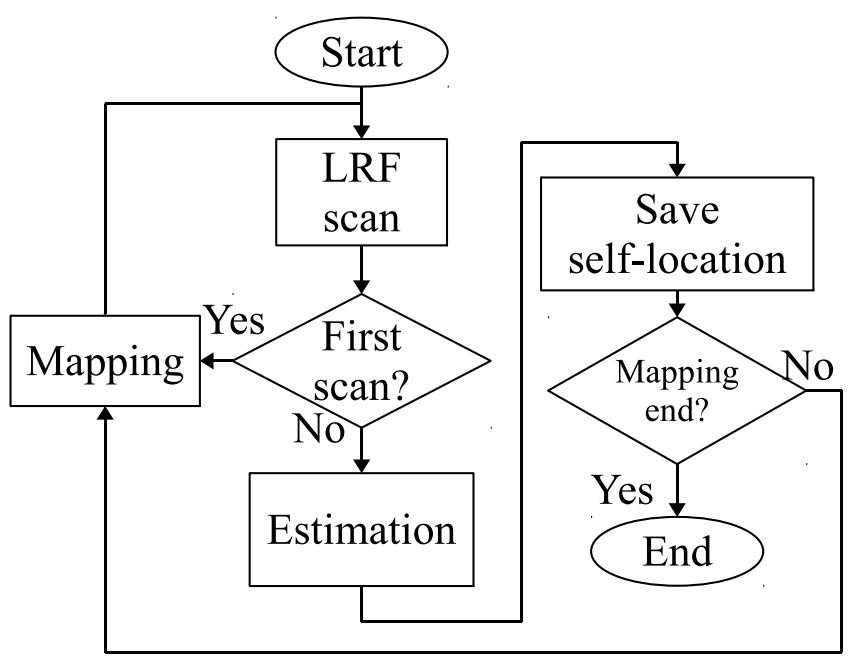

Fig. 4. System flow chart

of distance in $100 \mathrm{~mm}$ to $1000 \mathrm{~mm}$ and $\pm 30 \mathrm{~mm}$ in $1000 \mathrm{~mm}$ to $8000 \mathrm{~mm}$. Fig. 3 shows the position relation of the wheelchair and the LRF. The computer runs on Debian GNU/Linux 5.0. Its CPU is Athlon 64 X2 Dual-Core $5200+(2.6 \mathrm{GHz})$ and it has 4 GByte memories.

Fig. 4 shows a flowchart of localization and mapping. At first, this system receives the scan data of LRF. The first map is built from this scan data. At next scan, our system estimates the position and orientation of the wheelchair referring to the saved map.

\section{A. Map saving}

Typically, the environment map saving are of 2D type and $3 \mathrm{D}$ type. 3D type map is used for rescue robot which works in disaster site. This map requires a lot of data storage space. Our system support to navigates in daily life. Therefore, we selected 2D map in our research. This map is saved in gridded map which is called Occupancy Grid. The grid scale is $2 \mathrm{~cm} \times 2 \mathrm{~cm}=4 \mathrm{~cm}^{2}$ in this system. It can be used easily for estimation.

\section{B. Localization}

Estimation of location and orientation in this research is calculation of a movement distance and rotation angle at current position.

1) The conventional method: The most popular technique for state estimation is ICP. Let the position of the wheelchair be $x, y$ and the orientation be $\theta$. These variations are $\delta x, \delta y$ and $\delta \theta$. The conventional method minimize the this equation

$$
\sum_{i=0}^{n}\left(R \boldsymbol{p}+\boldsymbol{T}-\boldsymbol{p}^{\prime}\right)^{2}
$$

where $\boldsymbol{p}$ is an obstacle position vector of the current scan data, $\boldsymbol{p}^{\prime}$ is an obstacle position vector of the previous scan data and $n$ is number of feature points in scan data. $R$ is a rotational matrix defined by

$$
R=\left(\begin{array}{cc}
\cos \delta \theta & -\sin \delta \theta \\
\sin \delta \theta & \cos \delta \theta
\end{array}\right)
$$




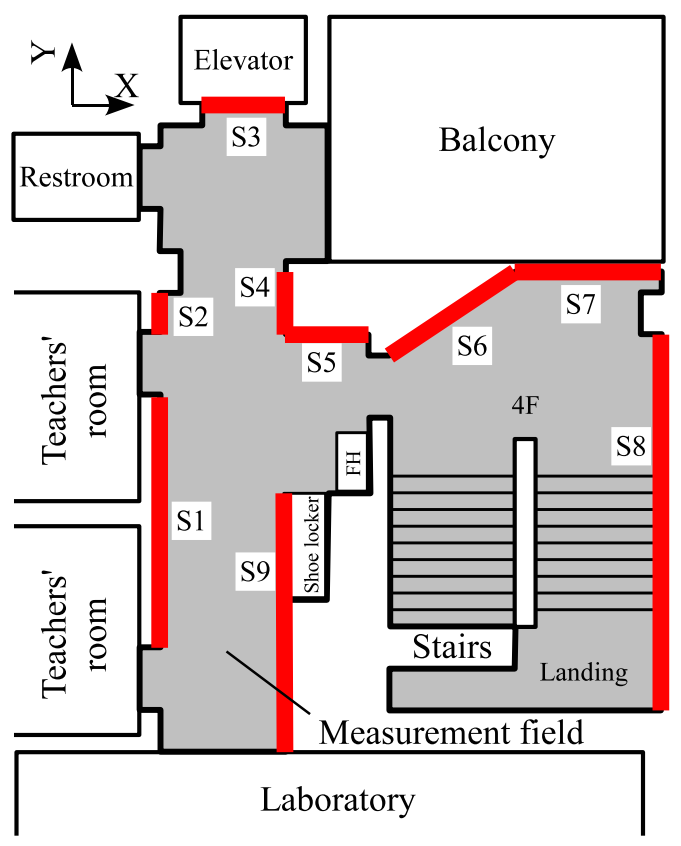

Fig. 5. Experiment environment

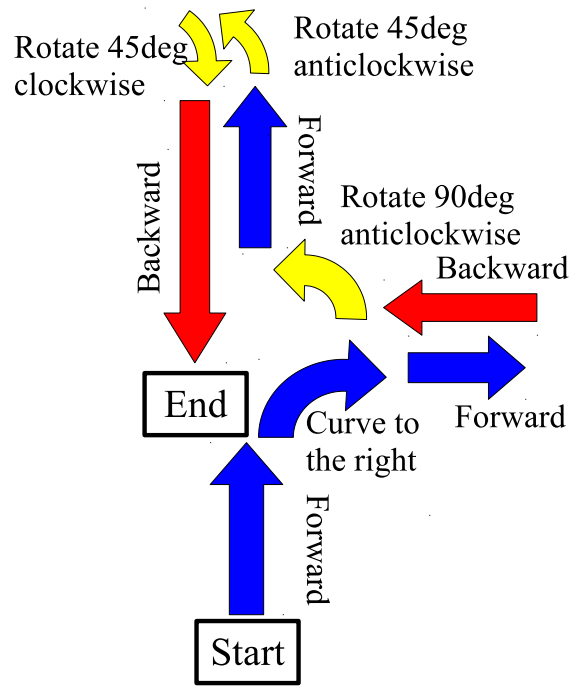

Fig. 6. Trajectory plan in experiment III

$\boldsymbol{T}$ is a translation vector which contains variations $\delta x$ and $\delta y$. The conventional method is nonlinear equation and needs feature of scan data.

2) The proposed method: Therefore we adopt Particle Swarm Optimization (PSO) algorithm which can calculate accurate values using iteration method. PSO is optimum solution in particles which simulated the location and the orientation of the wheelchair. These estimation values are the movement $\delta x, \delta y$ and rotation $\delta \theta$. These values put together into state vector $\boldsymbol{z}=[\delta x, \delta y, \delta \theta]^{T} \in R^{3}$ which has velocity vector $v \in R^{3}$. pbest $\in R^{3}$ is best solution of each particle and gbest $\in R^{3}$ is best solution of all particles. Our method

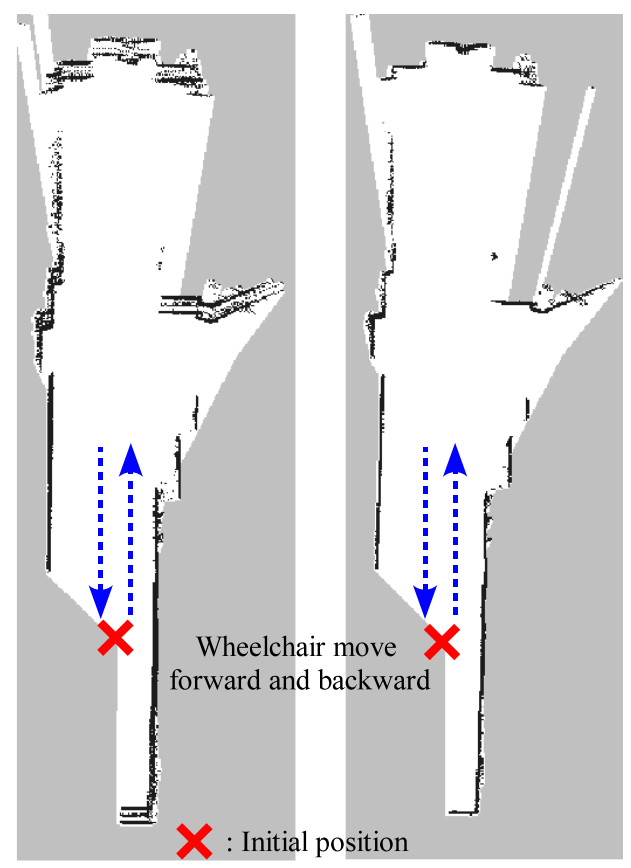

\section{Conventional Proposed method \\ method}

Fig. 7. Result of experiment I

minimize the evaluation function $f(\boldsymbol{z})$ which is shown

$$
f(\boldsymbol{z})=\frac{\text { Data }_{\text {valid }}(\boldsymbol{z})-\text { Data }_{f i t}(\boldsymbol{z})}{\text { Data }_{\text {valid }}(\boldsymbol{z})}
$$

where $\operatorname{Data}_{\text {valid }}(\boldsymbol{z})$ is the number of scan plots whose distance is over $300 \mathrm{~mm}$ and Data $\operatorname{Dat}_{\mathrm{f}}(\boldsymbol{z})$ is the number of scan plots which fit in the map. PSO algorithm is shown as follows

Step 0

Select number of particle $2<m \in R^{1}$, weight coefficients of particle $\omega, c_{1}, c_{2}$, and number of iterations $K_{\max }$. Set $k$ to 0 .

Step 1

Initialize state value of particles $\boldsymbol{z}_{i}^{0} i=1,2, \cdots, m$ and set velocity $\boldsymbol{v}_{i}^{0}=0$. Initialize pbest as pbest $=\boldsymbol{z}_{i}^{0} i=1,2, \cdots, m$. Set gbest $=$ $\operatorname{argmin} f\left(\boldsymbol{p b e s t}_{i}\right)$ for $i=1,2, \cdots, m$. Where $i$ is the number of particles and $k$ is the iteration number.

Step 2

Update state and velocity using random numbers.

$$
\begin{array}{cc}
\boldsymbol{v}_{i}^{k+1}=\omega \cdot \boldsymbol{v}_{i}^{k}+c_{1} \cdot \boldsymbol{R}_{i 1} \cdot\left(\text { pbest }_{i}-\boldsymbol{z}_{i}^{k}\right) \\
+c_{2} \cdot \boldsymbol{R}_{i 2} \cdot\left(\text { gbest }-\boldsymbol{z}_{i}^{k}\right) \\
\boldsymbol{z}_{i}^{k+1}=\quad & \boldsymbol{z}_{i}^{k}+\boldsymbol{v}_{i}^{k+1} i=1,2, \cdots, m
\end{array}
$$

where $\boldsymbol{R}_{i 1}, \boldsymbol{R}_{i 2}$ are random matrices which are shown $\boldsymbol{R}_{i 1}=\boldsymbol{R}_{i 2}=$ $\operatorname{diag}\left(\operatorname{rand}_{\mathrm{n}}(), \operatorname{rand}_{\mathrm{n}}(), \operatorname{rand}_{\mathrm{n}}()\right)$ and $\operatorname{rand}_{\mathrm{n}}()$ is random value which is between 0 and 1 . 


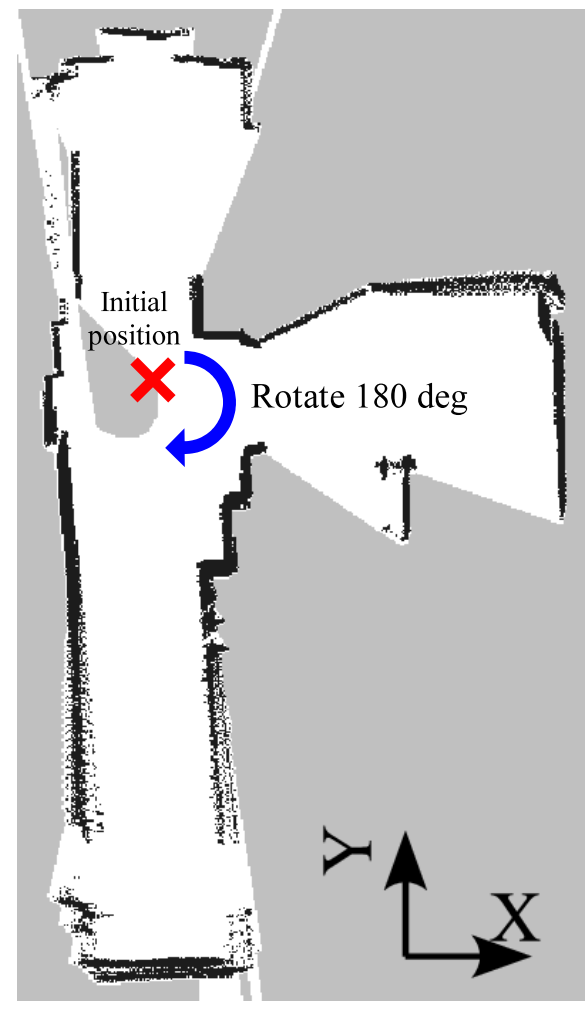

Fig. 8. Conventional method result in experiment II

Step 3

Update pbest $_{i}$ and gbest using evaluation function $f(\boldsymbol{z})$. Set pbest $_{i}=$ $\operatorname{argmin} f\left(\boldsymbol{z}_{i}\right)$ for $i=1,2, \cdots, m$ and Step 4 $\boldsymbol{g} \boldsymbol{b e s t}=\operatorname{argmin} f\left(\boldsymbol{p b e s t}_{i}\right)$ for $i=1,2, \cdots, m$.

If $k=K_{\max }$ or $f($ gbest $)<\epsilon$, finish the calculation. Otherwise return to step2. Where $\epsilon$ is coefficient of iteration end. In this paper, we set $\epsilon=0.05$.

Each parameter is shown as follows

- Number of particle: $m=40$

- Weight coefficients: $\omega=0.7, c_{1}=0.8, c_{2}=0.8$

- Number of iteration: $K_{\max }=30$.

These parameters are determined by trial and errors. Because state change between the previous and the current scanning of the wheelchair is very small, it is enough that initial state of particles are located within a limited area as follows. 10 particles are uniform located on $\mathrm{x}$-axis between $-500 \mathrm{~mm}$ to $500 \mathrm{~mm}$ which is shown as follows $\delta x=-500$ to $500, \delta y=$ 0 and $\delta \theta=0.10$ particles are uniform located on y-axis between $-500 \mathrm{~mm}$ to $500 \mathrm{~mm}$ which is shown as follows $\delta x=0, \delta y=-500$ to 500 and $\delta \theta=0.20$ particles have uniform rotation between $-50 \mathrm{deg}$ to $50 \mathrm{deg}$ which is shown as follows $\delta x=0, \delta y=0$ and $\delta \theta=-50$ to 50 .

\section{Unnecessary data deletion}

If some moving objects are in the environment, those positions change by each traveling. Therefore, we design unnecessary data deletion. Obstacles are deleted in $2 \mathrm{~m}$ distance

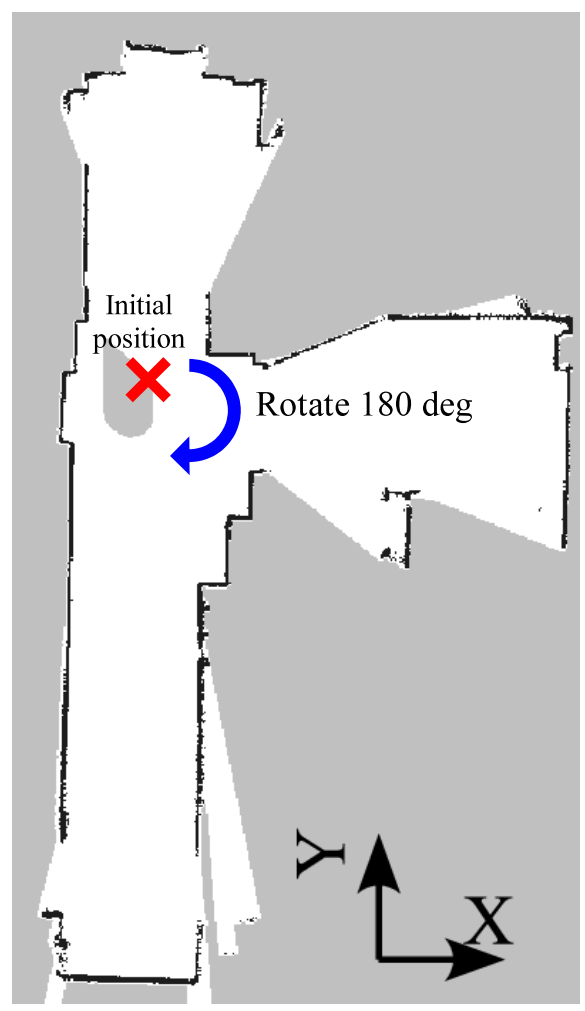

Fig. 9. Proposed method result in experiment II

by the LRF. The map will be updated with latest information by this deletion. The wheelchair can detect a door opening and closing.

\section{EXPERIMENTS}

In-door experiments under four situations are conducted for confirming availability of our method. The experimental environment is shown in Fig. 5. In Fig. 5, S1 to S9 are used to evaluate our mapping accuracy for third experiment. The wheelchair is pushed by an operator in these experiments. The conventional approach is Weiss's method [4], which uses cross correlation by the previous and current scan data for estimation.

In all experiments, the initial position and orientation of the wheelchair is $x=0 \mathrm{~mm}, y=0 \mathrm{~mm}$ and $\theta=0 \operatorname{deg}$ at the first measurement point. The first experiment is for the evaluation of estimation accuracy in Y-axial direction movement without rotation. The wheelchair moves forward by $2 \mathrm{~m}$ and return to initial position. Scanning data are taken by each $10 \mathrm{~cm}$ movement. The second experiment is for the evaluation of estimation accuracy in rotational movement. The wheelchair rotates clockwise by $180 \mathrm{deg}$ at initial position in about $20 \mathrm{sec}$. Scanning data are taken each about $0.2 \mathrm{sec}$. The third experiment is for the evaluation of localization and mapping accuracy in the environment. The wheelchair moves across the track as shown in Fig. 6 and takes scanning data each about $0.2 \mathrm{sec}$. We measure processing time for estimation. The forth experiment is for the confirmation of mapping and unnecessary data deletion about the dynamic environment including the wandering persons. 


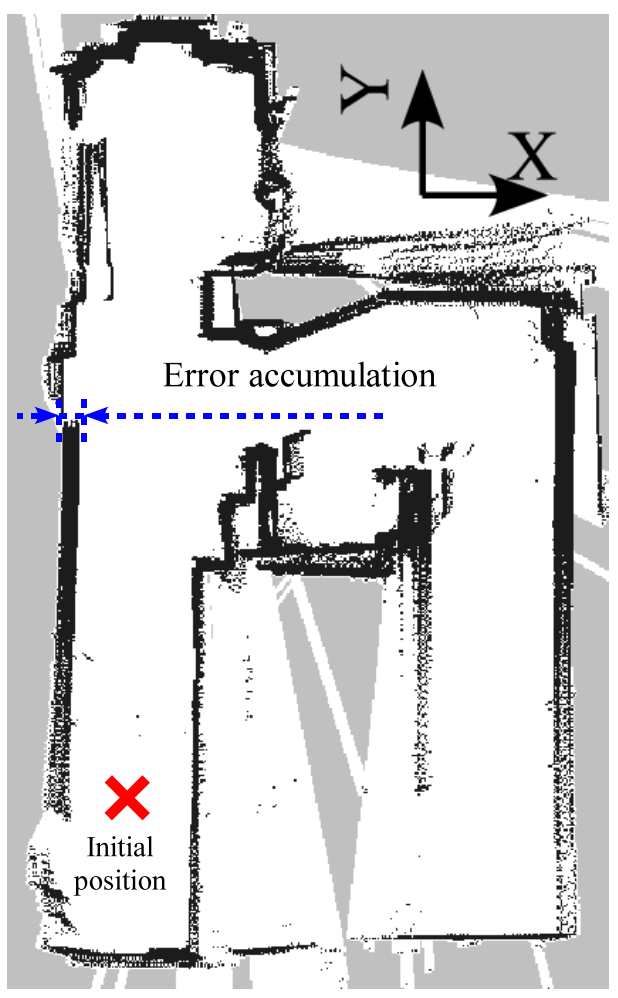

Fig. 10. Conventional method result in experiment III

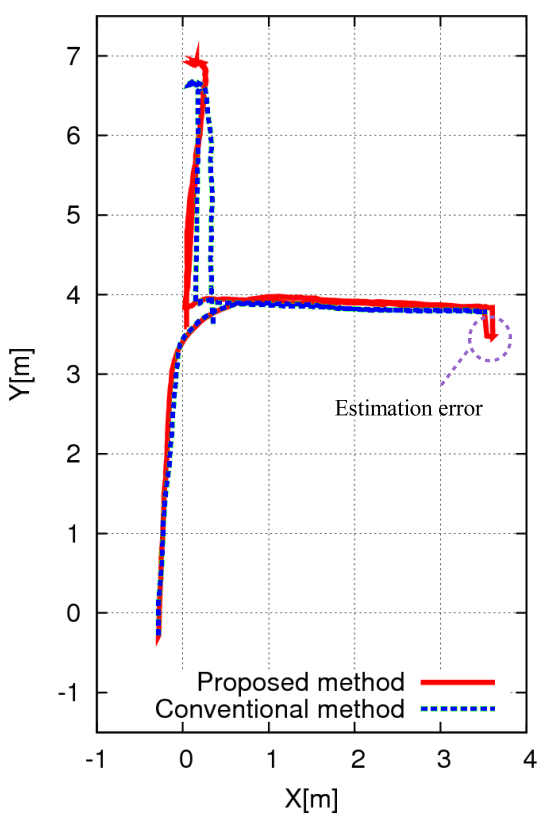

Fig. 12. Trajectry results in experiment III

The wheelchair remains stationary and several persons move in this environment. Scanning data are taken for $20 \mathrm{sec}$ at intervals of approximately $0.2 \mathrm{sec}$.

\section{A. Experimental results}

Colors of these maps following meanings.

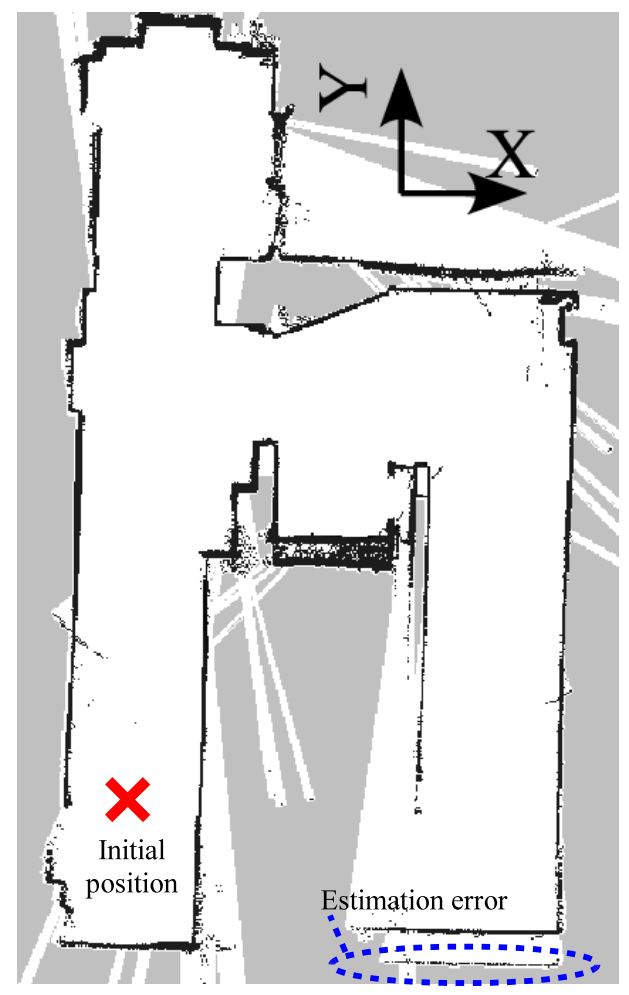

Fig. 11. Proposed method result in experiment III

TABLE I

COMPARISON BETWEEN CONVENTIONAL AND PROPOSED METHOD MOVEMENT IN EXPERIMENT I

\begin{tabular}{ccc}
\hline & Conventional & Proposed \\
\hline Forward average [mm] & 98.50 & 100.7 \\
\hline Forward dispersion [mm] & 25.14 & 14.24 \\
\hline Forward standard deviation [mm] & 5.014 & 3.774 \\
\hline Forward position [mm] & 1970 & 2013 \\
\hline Forward error [mm] & 30.03 & -13.36 \\
\hline Backward average [mm] & -99.76 & -101.0 \\
\hline Backward dispersion [mm] & 23.71 & 28.16 \\
\hline Backward standard deviation [mm] & 4.869 & 5.307 \\
\hline Backward position [mm] & -25.16 & -11.84 \\
\hline
\end{tabular}

TABLE II

COMPARISON BETWEEN CONVENTIONAL AND PROPOSED METHOD ROTATION IN EXPERIMENT II

\begin{tabular}{ccc}
\hline & Conventional & Proposed \\
\hline Orientation[deg] & -173.0 & -179.6 \\
\hline Error[deg] & -7.0 & -0.4 \\
\hline
\end{tabular}

- Gray is Unknown Area.

- Black points are Obstacles.

- White is Measured Area.

Fig. 7 shows a mapping result of the first experiment by each method. The average, dispersion, standard deviation, position and estimation errors of each method are shown in Table I. In Fig. 7, the conventional method has errors which is about $240 \mathrm{~mm}$ in $\mathrm{Y}$ axis direction. On the other hand our method has high accuracy because each step error is small and final position is near the correct value. Fig. 8 
TABLE III

COMPARISON BETWEEN MEASURED LENGTH AND ESTIMATION LENGTH IN EXPERIMENT III

\begin{tabular}{ccccc}
\hline Sides & Measured $[\mathrm{mm}]$ & Estimation $[\mathrm{mm}]$ & Error $[\mathrm{mm}]$ & Error ratio [\%] \\
\hline S1 & 4490 & 4482 & -8 & -0.17 \\
\hline S2 & 590 & 601 & 11 & 1.92 \\
\hline S3 & 800 & 780 & -20 & -2.47 \\
\hline S4 & 748 & 781 & 33 & 4.42 \\
\hline S5 & 510 & 500 & -10 & -1.88 \\
\hline S6 & 1320 & 1405 & 85 & 6.41 \\
\hline S7 & 2170 & 2040 & -130 & -5.99 \\
\hline S8 & 6710 & 6603 & -107 & -1.59 \\
\hline S9 & 4445 & 4422 & -23 & -0.51 \\
\hline
\end{tabular}

TABLE IV

RESULT OF PROCESSING TIME IN EXPERIMENT III

\begin{tabular}{cc}
\hline & Processing time [sec] \\
\hline Average & 0.066 \\
\hline Minimum & 0.036 \\
\hline Maximum & 0.128 \\
\hline
\end{tabular}

and Fig. 9 show mapping results of the second experiment. Some errors occur in the conventional method and the result of our method has few errors as shown in Table II. The difference between the correct value and the estimated value using the comparative method is $7 \mathrm{deg}$ by Table II. Our method has high accuracy estimation of rotation. Fig. 10 and Fig. 11 show mapping results of the third experiment. Fig. 12 shows trajectory results of the same experiment. Table III is comparison between measured length and estimated length by our method. From Fig. 11, it is confirmed that our method has high accuracy mapping. However the estimation failure is observed at one point. In Table IV, the processing time of our method suffices to the navigation. Considering that the maximum operating speed of the wheelchar is 3 $\mathrm{km} / \mathrm{h}$, our method with a estimation period of about 100 msec is competent completely. Fig. 13 shows the picture of unnecessary data deletion in fourth experiment. The wandering person moves in the experimental environment as indicated in Fig. 13. Check data which temporary stored can confirm whether it is the trace of the wandering person. After the movement of the object, unnecessary data is deleted from the environment map. From all experimental results it is observed that our method has high accuracy estimation and high processing speed.

\section{CONCLUSION AND FUTURE WORK}

We proposed high accuracy and fast estimation method for localization and mapping. Our environmental map is saved in gridded map. The position and orientation are estimated by using particles using PSO algorithm. We confirmed accuracy and short processing time of our method by some experiments. The experimental results clearly show that our method has higher accuracy than the conventional method. Future works are generation of a safety path and controller design for autonomous navigation.

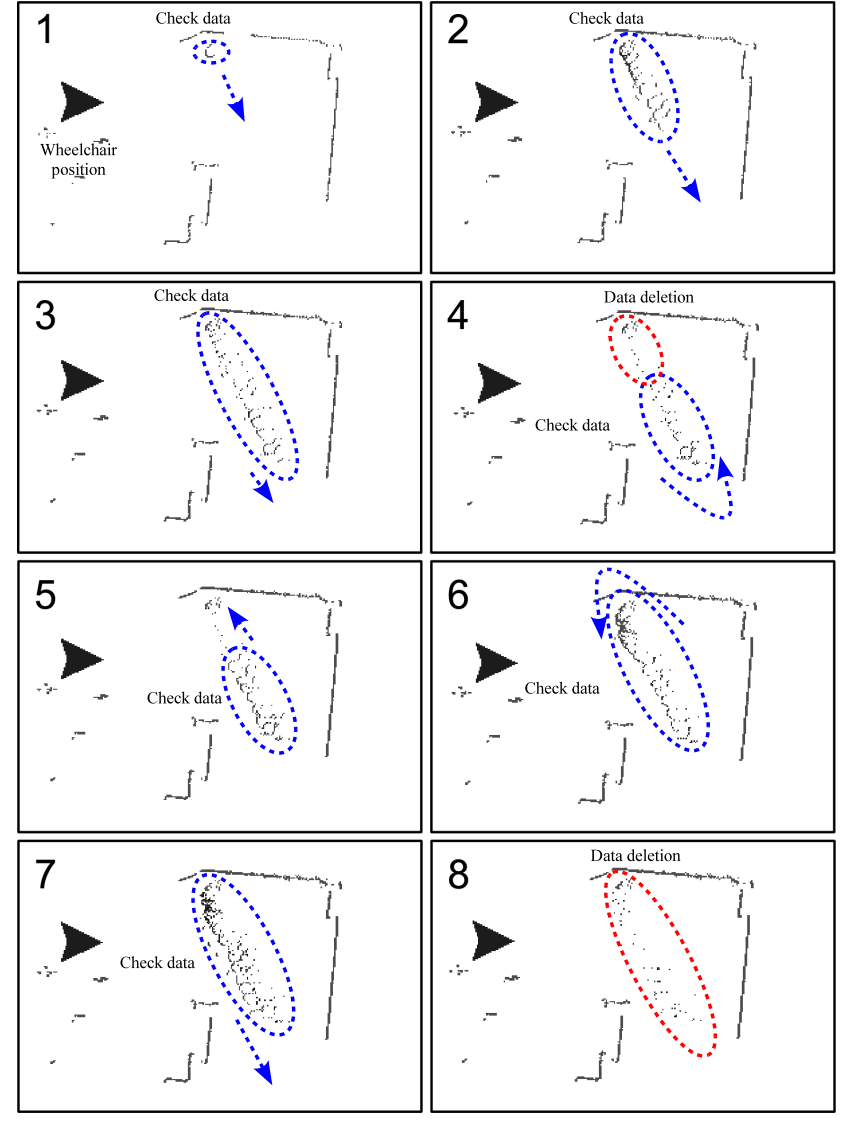

Fig. 13. Result of experiment IV

\section{REFERENCES}

[1] O. Matsumoto, K. Komoriya, T. Hatase, T. Yuki and S. Goto: "Intelligent Wheelchair Robot "TAO Aicle"”, Service Robot Applications, pp.55-70, 2008.

[2] M. Montemerlo, S. Thrun, D. Koller and B. Wegbreit: "FastSLAM: A Factored Solution to the Simultaneous Localization and Mapping Problem", Proceedings of the AAAI National Conference on Artificial Intelligence, pp.593-598, 2002.

[3] K. Ohno, K. Nagatani and H. Akiyama: "Mapping for Rescue Robots", Jounal of the Robotics Society of Japan, Vol.28, No.2, pp.169-172, 2010.

[4] G. Weiss, G. Wetzler and E. V. Puttkamer: "Keeping Track of Position and Orientation of Moving Indoor Systems by Correlation of RangeFinder Scans", Proc. of IROS, pp.595-601, 1994.

[5] Paul J. Besl and Neil D. McKay: "A Method for Registration of 3-D Shapes", IEEE Trans. on PAMI, Vol.14, No.2, pp.239-256, 1992.

[6] S. Rusinkiewica and M Levoy: "Efficient Variants of the ICP Algorithm”, International Conference on 3-D Ditital Imagin and Modeling, pp.145-152, 2001.

[7] H. Yoshitaka, K. Hirohiko, O. Akihisa and Y. Shin'ichi: "Mobile Robot Localization by Scan Matching using Laser Reflectance Intensity of the SOKUIKI Sensor ”, Robotics and Mechatronics Conference 2006, 2P1-C29, 2006.

[8] Y. Tsukada, T. Otsuka, A. Watanabe, Y. Uchimura: "Self-Localization for Autonomous Outdoor Mobile Robot", Technical Meeting Record on Industrial Instrumentation and Control, Vol.1, pp.5-9, 2011.

[9] J. Kennedy and R. C. Eberhart: "Particle swarm optimization", Proc. of IEEE International Conference on Neural Networks, pp. 1942-1948, 1995.

[10] K. Okada and Y. Fujimoto: "Simultaneous Localization and Mapping Method without odometry information", Technical Meeting Record on Industrial Instrumentation and Control, Vol.1, pp.29-34, 2011. 